\title{
Significance of Transverse Sinus Stenosis in Idiopathic Intracranial Hypertension Patients Using Contrast Enhanced Magnetic resonance Venogram
}

\author{
Dr.M.Ramesh MD [RD] ${ }^{1,}$ Dr .S.Babu Peter MD[RD],DNB ${ }^{2,}$ \\ Dr. N.Kailasanathan MD[RD],DMRD ${ }^{2}$, \\ ${ }^{1}$ Assistant Professor Of Radiology, Government Medical College Omandurar Estate ,Chennai. \\ ${ }^{2}$ Professor ,Barnard Institute Of Radiology,Madras Medical College, Chennai, \\ ${ }^{3}$ Professor And Director ,Barnard Institute Of Radiology,Madras Medical College, Chennai
}

\begin{abstract}
Background : Incidence of IIH is among population in united states is 1/10000 in general population, increasing to 1.6- 3.5 per 1lakh population in females and further increasing to 8 to 20 per 1lakh population in females of reproductive age group and obese [4] . Quincke first described idiopathic intracranial hypertension in 1893. Later in 1937 diagnostic criteria was first put forth by Dandy which was further modified by Smith in 1985 as Modified Dandy criteria. With the advent of MRI, few MR features like partial empty sella, prominent perioptic subarachnoid space, tortuous optic nerve, flattening of posterior globe of eye and papillary optic nerve protrusions, small and pinched ventricles has a sensitivity around 50 to $75 \%$.The observation of narrowed transverse-sigmoid sinus conduit in IIH noted with high sensitivity and specificity .

Methods: In both cases and controls contrast enhanced MRvenogram is done by time resolved method after acquiring routine MR sequences, then by post processing, subsegmental MIP image [volume MIP image of posterior part of superior sagittal sinus, bilateral transverse and sigmoid sinus] is derived. Then posterior part superior sagittal sinus diameter is compared with stenosed transverse sinus for percentage of stenosis and scoring is given. A score less than 5 out of 8 is considered positive for case

Results : Combined conduit scoring for transverse sinus narrowing is more sensitive[90\%] than other MRI features [50\%-75\% ], whereas specificity is comparable between them [around 90\%]

2D TOF MRI showed more false positivity due to signal loss in slow flow and narrowed sinues

Conclusion: Combined conduit scoring for transverse sinus stenosis is more sensitive method compared to other MR features of IIH like partial empty sella, prominent perioptic subarachnoid space, tortuous optic nerve, flattening of posterior globe of eye and papillary optic nerve protrusions, pinched ventricles .Contrast enhanced MR venogram is more superior to 2DTOF MRI. Invasive procedure of Lumbar puncture for CSF manometry can be replaced by CCS scoring for transverse sinus stenosis and other MR features.CCS scoring can also be used for follow up of cases instead of repeat lumbar punctures

Keywords: MRI-Magnetic resonance imaging CEMRV-Contrast enhanced magnetic resonance venogram IIH - Idiopathic intracranial hypertension CCS-Combined conduit scoring 2D TOF MRV-2 dimensional time of flight magnetic resonance venogram Subsegmental MIP image Partial empty sella.

prominent perioptic subarachnoid space Tortuous optic nerve, Flattening of posterior globe of eye Papillary optic nerve protrusions, ,Pinched ventricles
\end{abstract}

\section{Introduction}

$\mathrm{IIH}$ is fast emerging because of the increasing incidence of obesity and one of the cause of treatable blindness causing great morbidity .There are no acceptable and highly sensitive imaging criteria . Modified Dandy criteria has not been modified since 1985,after the emergence of MRI . Invasive procedure like csf opening pressure measurement is mandatory to confirm intracranial hypertension .A non invasive imaging criteria which is highly sensitive enough to replace invasive procedure is required. Previous Studies based on transverse sinus narrowing as the criteria for IIH has showed rewarding results. CEMRV is considered superior to $2 \mathrm{D}$ TOF MR venogram and phase contrast MRvenogram .

3 major theories proposed for etiology of IIH ,but most accepted theory is venous outflow obstruction [4] various secondary causes for intracranial hypertension are medical disorder,medications, endocrine disturbances, venous thrombosis [10].A typical case of idiopathic intracranial hypertension is a obese women in the child bearing age group [10]. The classic symptoms are head ache, obscuration of vision, pulsatile tinnitus, double vision ,photopsia and rarely blindness. Signs of IIH include papilledema, preservation of central vision, diplopia commonly due to 6 th nerve palsy [6] 


\section{Modified Dandy criteria}

1. Signs and symptoms of increased intracranial pressure

2. No localizing neurologic signs, with exception of unilateral or bilateral VI nerve paresis.

3. CSF can show increased pressure, without cytologic or chemical abnormalities .

4. Small symmetric ventricles must be demonstrated [10] .

2D TOF MR venogram is based on the intravascular flow producing signal. so artifacts results from slow intravascular flow ,in plane flow, complex blood flow patterns and post processing.

Phase contrast MR venogram depends on velocity induced phase shifts on moving spins. It requires long imaging times and proper estimation of blood flow velocities to avoid signal loss due to turbulence and intravoxel dephasing [12]. Contrast enhanced 3D MR venogram is the best among imaging studies which shortens the T1 relaxation time and it is flow independent. The image acquisition time should be optimized for good opacification with power injectors and bolus tracking methods .

Time resolved contrast MR venogram is a technique wherein the acquisition is started immediately after bolus injection of contrast and the sequence is repeated multiple times [13,14,15]

Treatment options available are medical and surgical methods. Medical management includes weight reduction, oral acetazolamide and steroids in resistant cases . Surgical management is indicated in medical management resistant cases. Optic nerve fenestration in indicated in patients with severe papilledema and less ICT symptoms. Lumboperitoneal shunting is indicated in patients with severe ICT symptoms. Dural venous sinus stenting in indicated in transverse sinus stenosis with pressure gradient above 10mm[27] [ 28]

\section{Aim Of The Study}

To evaluate prevalence of bilateral transverse sinus stenosis using dynamic contrast enhanced magnetic resonance venogram in idiopatic intracranial hypertension

\section{Materials And Methods}

Study Population - Our study included 64 persons. The cases and controls were referred from Neurology department. The observer is blinded about whether a case or control is referred for MR examination. A case of idiopathic intracranial hypertension is diagnosed based on CSF opening pressure more than $250 \mathrm{~mm}$ of water in our study

Type of study- Case control study

\section{case}

Inclusion criteria :

- CSF opening pressure greater than $250 \mathrm{mms}$ of $\mathrm{H} 2 \mathrm{O}$ with normal CSF constituents and normal imaging study ;

\section{Exclusion criteria}

- Pregnant and breast feeding women

- Severe hypersensitivity or previous allergic reactions

- Critically ill patients

- Known cause of intracranial hypertension identified like drugs, systemic disorders

- Known case of intracranial space occupying lesion ,as every case or control screened with CT brain

- Previous history of sinus thrombosis

- Two case is excluded from our study as they showed sagittal and distal transverse sinus thrombosis using MRI and dynamic contrast Enhanced MR venogram

- One case excluded because post contrast study showed diffuse enhancement of leptomeninges suggestive of meningitis

- One case is excluded from the study as the patient taking steroids irregularly for rheumatoid arthritis

\section{Controls}

- Patients with clinical symptoms of IIH like headache, tinnitus,blurring of vision for whom CSF pressure is less than $250 \mathrm{cms}$ of water

- Patients who was fixed for MRI brain with contrast for some other reasons for whom CSF manometry is done after consent and found csf pressure within limits

\section{Blinding}


Cases and controls were referred from the department of Neurology,Madras medical college. The Neurologist performing CSF pressure is blinded of the MRI features of the patient, only the neurologist knows whether a referred person is case or control

Radiologist interpreting the MR images were blinded of the clinical information and CSF pressure . After completing the study, the results were discussed by radiologist and neurologist together on case by case basis and results were tabulated

\section{Age Range}

Cases - Age ranged from 17 years to 55 years

Controls- Age ranged from 22 years to 60 years

Sex

Cases - 28 females and 2 males

Controls- 23 females and 7 males

\section{Materials}

- $\quad 1.5$ tesla super conducting Magnetom Siemens

- Standard head coil

- Gadolinium dimeglumine $10 \mathrm{ml}$, saline bolus $20 \mathrm{ml}$

\section{Methods}

1. Initially MRI routine sequences of brain is obtained .

2. $2 \mathrm{D}$ TOF MR venogram acquired in axial plane with following parameters TR- 30,TE-6.5 ,FOV 145X145, 256X256,BW - 292,Flipangle -60

3. Contrast enhanced magnetic resonance venogram done using SPGR sequence, the sequence parameters are TR 3.6, TE- 1.4,slip angle-30,slice thickness $2.5 \mathrm{~mm}$, fov- 292x360. 187x 256, BW-348.

Coronal precontrast and post contrast SPGR sequence are obtained after 10ml of gadolinium with $20 \mathrm{ml}$ saline chase .Post contrast sequence is acquired repeatedly for 4-5 times, then the best post contrast image is subtracted with the pre contrast image and then mipped to get 3D MIP image, then unnecessary part of the sinus are removed leaving behind the posterior half of superior sagittal sinus, transverse sinus and sigmoid sinus [subsegmental MIP image ] for proper assessment of the stenosis

\section{Combined conduit score using subsegmental MIP image}

In our study the distal sagittal sinus diameter is measured and compared with narrowed part of the sigmoid and transverse sinus conduit .

If there is a flow gap in the sigmoid transverse conduit, then the score is 0 ,

If the narrowed transverse diameter is between $1-25 \%$ of the distal sagittal sinus then the score is 1 ,

If the narrowed sinus diameter is between $26 \%-50 \%$ then a score is 2 ,

If the narrowed diameter is between $51 \%-75 \%$ then the score is 3

If the narrowed diameter of the transverse sinus is between $76 \%-100 \%$ then the score is 4 and it is considered normal.

So the total score is 8 which is called combined conduit score

In our study we considered a score less than 5 as significant

CSF opening pressure is measured in lateral decubitus position using lumbar puncture 20-22 $\mathrm{g}$ spinal needle and a score more than $250 \mathrm{cms}$ of water is considered as positive

\section{Results}

Statistical analysis for sensitivity, specificity, test for significance was done for the acquired data. Out of 30 cases , 27 cases had significant bilateral stenosis with a combined conduit score less than 5, whereas in controls only 4 persons had combined conduit score less than 5. Hence sensitivity and specificity of is $90 \%$ and $86 \%$ respectively . The positive and negative predictive value of the test is $87 \%$ and $89 \%$

\begin{tabular}{|l|l|l|}
\hline & cases & controls \\
\hline Ccs $<5$ & 27 & 4 \\
\hline Ccs $>$ or $=5$ & 3 & 26 \\
\hline total & 30 & 30 \\
\hline
\end{tabular}

Sensitivity - $\quad 90 \%$

Specificity $\quad-\quad 86 \%$ 
PPV $\quad-87 \%$

NPV $\quad-\quad 89 \%$

Test of significance using chi square test was done using combined conduit score in control and cases

There is significant correlation between csf pressure and combined conduit score and the scoring using contrast enhanced venogram is correlating well with $\mathrm{p}$ value less than 0.001 .On comparison of CCS score with other MRI features using chisquare test, $\mathrm{p}$ value of .014 is obtained with less than $5 \%$ significance suggesting statistically significant degree of correlation in which the combined conduit score has more statistically more useful than other MRI features

Comparision of various MR features in idiopathic intracranial hypertension

\begin{tabular}{|l|l|c|l|l|}
\hline Mri features & cases & Sensitivity\% & controls & Specificity\% \\
\hline Ccs $<5$ & 27 & 90 & 4 & 86.67 \\
\hline Patulous optic nerve sheath & 20 & 66 & 3 & 90 \\
\hline Small symertrical ventricles & 15 & 50 & 2 & 93.33 \\
\hline flattening of the posterior sclera & 18 & 60 & 2 & 93.33 \\
\hline partially empty sella & 22 & 73 & 3 & 90 \\
\hline
\end{tabular}

From the above data it is clear that the sensitivity of combined conduit score is $90 \%$ and it is more than the sensitivities of other MR features The specificity of various MR features in the diagnosis of IIH is around $90 \%$ for all features . chisquare test comparing between MR venogram and 2D TOF venogram in cases and control showed a $\mathrm{P}$ value of 0.22 suggesting significant difference between CEMRV and 2D TOF

\section{Discussion}

From the results it obvious that combined conduit scoring of bilateral sigmoid and transverse sinus stenosis using contrast enhanced MR venogram is highly sensitive test in diagnosing idiopathic intracranial hypertension. Three cases which showed combined conduit score of 5 were in the borderline stage and showed false negative values and all such cases had borderline elevation in pressure between 250 to $300 \mathrm{mms}$ of water. The possibilities for false negatives may be because of imaging error or csf pressure monitoring error .

The specificity of the test is $86 \%$ with four of the patients has higher combined conduit score . Again in all four cases the ccs score is borderline with a score of 4 in all four cases. In all these 4 controls only one side is involved and medial transverse sinus is involved, whereas in cases, both transverse sinus either symmetrically or asymmetrically and lateral aspect of the transverse sinus is involved

In our study using 2D TOF MRV, we observed significant false positive values in controls with 14 out of 30 controls showing score less than 5 possibly due to due to in plane flow error, slow flow. In nearly all the cases which showed signal gaps, contrast enhanced venogram showed some flow

Out of 30 cases , 3 cases showed pressure more than $400 \mathrm{mms}$ of water . In those cases , 2 cases showed a score of $2 / 8$ and one case showed a score of 3/8. From this it is clear that as ccs score decreases as the csf pressure increases and hence can be used for follow up .

Other known MRI features of IIH had sensitivities between 50 to $75 \%$., whereas CCS scoring is highly sensitive about $90 \%$

The specificity of various MRI features and CCS is comparable and above $90 \%$

We found papillary optic nerve protrusion has very low sensitivity[ 13.3\% ] and high specificity [ $100 \%$ ]

In our study obesity is correlated well with idiopathic intracranial hypertension with 27 out of 30 cases had BMI more than or equal to 28

\section{Conclusion}

- Contrast enhanced MR venogram with combined conduit score is more sensitive with sensitivity of about $90 \%$, compared to other MRI features

- Contrast MR venogram is superior to 2D TOF MRvenogram by reducing False positives

- Modified dandy criteria which include pinched ventricle is less sensitive, hence the criteria should be modified with CCS scoring and other MR features

- Invasive CSF manometry can be avoided and replaced by CCS scoring, atleast in high risk patients and in follow up after treatment

\section{References}

[1]. Jacobson DM, Friedman DI,. Idiopathic intracranial hypertension. J Neuroopthalmol. Jun 2004;24(2):138-45.

[2]. Newman NJ,Miller NR. Pseudotumor cerebri In:. Walsh and Hoyt 's clinical Neuroopthalmolog. Vol 1. 5th ed. 1999:523-38.

[3]. Durcan FJ, Wall M, Corbett JJ. The incidence of pseudotumor cerebri. Population studies in Iowa and Louisiana. Arch NeurolArch Neurol. Aug 1988;45(8):875-7 
[4]. AJNR .A2404 Pseudotumor Cerebri: Brief Review of Clinical Syndrome and Imaging Findings A.J. Degnan ,L.M. LevyRadioGraphics doi: 10.1148/rg.26si055174 October 2006 s, 26, S19-S41. Imaging of Cerebral Venous Thrombosis: Current Techniques, Spectrum of Findings, and Diagnostic Pitfalls

[5]. A JNR 2000 21: 74-78 Cerebral MR Venography: Normal Anatomy and Potential Diagnostic Pitfalls

[6]. Neurology January 8, 2002 vol. 58 no. 1 5-6 ,doi: 10.1212/WNL.58.1.5, Idiopathic intracranial , An answer to, "the chicken or the egg?

[7]. 2011 Aug;152(2):163-9. Epub 2011 Jun 21.Update on idiopathic intracranial hypertension. Department of Ophthalmology, Emory University, Atlanta, Georgia, USA

[8]. RWH walker Department of Neurology, Barts and the London NHS Trust, The Royal London Hospital, Whitechapel, London E1 1BB,UK J Neurol Neurosurg Psychiatry 2001;71:1-7, Idiopathic intracranial hypertension: any light on the

[9]. mechanism of the raised pressure?

[10]. Neurology 2002;59;1492 ,Deborah I. Friedman and Daniel M. Jacobson

[11]. Diagnostic criteria for idiopathic intracranial hypertension DOI 10.1212/01. WNL.0000029570.69134.1B Neurology 2002;59;1492

[12]. Case Reports in Otolaryngology Volume 2012 (2012), Article ID 814696, 8 pages ,doi:10.1155/2012/814696Case Report ,Benign Intracranial Hypertension: A Diagnostic Dilemma ,Stephanie and K. Gary Y. Shaw, Million Kansas City University of Medicine and Biomedical Sciences, Kansas City, MO 64086, USA

[13]. 2005 Sep;252(9):1021-5. 2005 Mar 7.Comparison of different MR venography techniques for detecting transverse sinus stenosis in idiopathic intracranial hypertension,. Institute of Neurological Sciences, National Research Council, Mangone (Cosenza), Italy.

[14]. Magnetic Resonance Venography Of Intracranial Venous Diseases

[15]. Jiing-Feng Lirng*Department of Radiology, Taipei Veterans General Hospital, and National Yang-Ming University School of Medicine, Taipei, Taiwan, R.O.C.

[16]. Heiserman JE, Dean BL, Hodak JA, , Bird CR, , Fram EK ,Flom RA.,Drayer BP Neurologic complications of cerebral angiography. AJNR Am J Neuroradiol 1994;15:1401-7.

[17]. Agid R, , Scott JN, Farb RI,Shelef I ,Imaging of the intracranialvenous system. Neurologist 2008;14:12-22.

[18]. Intracranial Venous System: Gadolinium-enhanced Three-dimensional MR Venography with Auto-triggered Elliptic Centricordered Sequence-Initial Experience From the Department of Medical Imaging, Division of Neuroradiology, Toronto Western Hospital, University of Toronto, December 2, 2002, doi: 10.1148/radiol.2261020670 January 2003 Radiology, 226, 203-209

[19]. High-Spatial-Resolution Contrast-enhanced MR Angiography of the Intracranial Venous System with Fourfold Accelerated Twodimensional Sensitivity Encoding, MD From the Magnetic Resonance Laboratory, Department of Radiology, Mayo Clinic College of Medicine ,doi: 10.1148/radiol.2433060819 June 2007 Radiology, 243, 853-861

[20]. Radiology rsna.org doi: 10.1148/radiol.2343031956 March 2005 Radiology, 234, 901-908. Three-dimensional Venography of the Brain with a Volumetric Interpolated Sequence, From the Department of Radiology, Onze Lieve Vrouw Hospital Aalst, Moorselbaan 164, 9300 Aalst, Belgium

[21]. Journal of Neurology, Neurosurgery \& Psychiatryjnnp.bmj.com ,J Neurol Neurosurg Psychiatry 2004;75:621-625 doi:10.1136/jnnp.2003.021006 .MR venography in idiopathic intracranial hypertension: unappreciated and misunderstood

[22]. DOI 10.1212/01.WNL.0000066683.34093.E2,Neurology 2003;60;1418

[23]. R. I. Farb, I. Vanek, J. N. Scott, et al. Idiopathic intracranial hypertension : The

[24]. prevalence and morphology of sinovenous stenosis

[25]. Cephalalgiacep.sagepub.com,doi: 10.1111/j.1468-2982.2009.01896.x Cephalalgia February 2010 vol. 30 no. 2 145-151.The upper limit of normal CSF opening pressure is related to bilateral transverse sinus stenosis in headache sufferer

[26]. June 2008, Volume 255, pp 807-812 .Bilateral transverse sinus stenosis and idiopathic intracranial hypertension without papilledema in chronic tension-type headache,

[27]. American Journal of Neuroradiology, doi: 10.3174/ajnr.A2575 AJNR 2011 32: 1408-1414 Interventional .Transverse Sinus Stenting for Idiopathic Intracranial Hypertension: A Review of 52 Patients and of Model Predictions 24 American Journal of Neuroradiology, doi: 10.3174/ajnr.A2463 AJNR 2011 32: 1021-1029 .MR Imaging Findings in Patients with Secondary Intracranial Hypertension

[28]. Journal of Child Neurology . doi: 10.1177/0883073809338874 J Child Neurol March 2010 vol. 25 no. 3 294-299 Magnetic Resonance Imaging Changes in Idiopathic Intracranial Hypertension in Children

[29]. 2006 Aug;48(8):521-7 2006 May 16.Idiopathic intracranial hypertension: the validity of cross-sectional neuroimaging signs

[30]. Arch Dis Child 1998;78:89-94 Diagnosis and management of benign intracranial

[31]. Hypertension ,D Soler, , P Bullock, , R O Robinson, T Cox, D M Calver

[32]. AJNR , doi: 10.3174/ajnr.A2575 AJNR 2011 32: 1408- -1414.Transverse Sinus Stenting for Idiopathic Intracranial Hypertension: A Review of 52 Patients and of Model Predictions ,

\section{Annexur Normal Anatomy Superior dural sinuses}




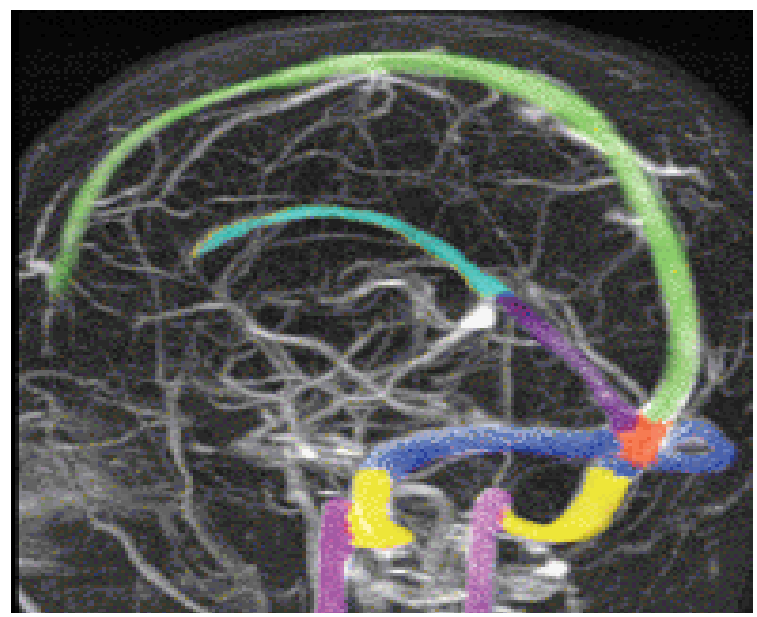

Contrast enhanced venogram in MIP format shows superior sagittal sinus in green, transverse sinus in blue, sigmoid sinus in yellow,jugular veins in light purple, straight sinus in dark purple,inferior sagittal sinus in light blue, torcula herophili in yellow colour

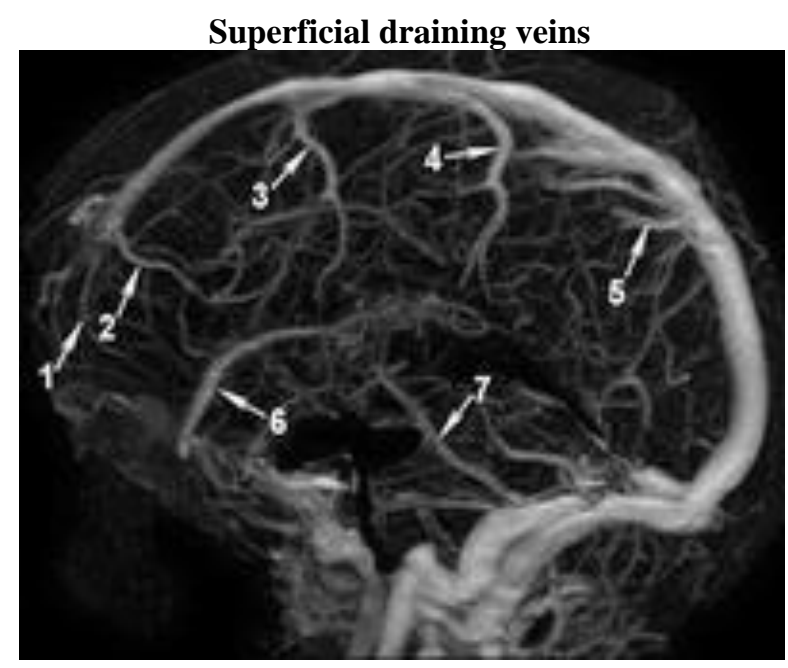

MR venogrpahic image image after removing deep sinus

1- Frontopolar 5 - anterior parietal vein

2- Anterior frontal 6 superficial middle cerebral vein

3- Posterior frontal 7 labbe vein

4- Vein of trolard

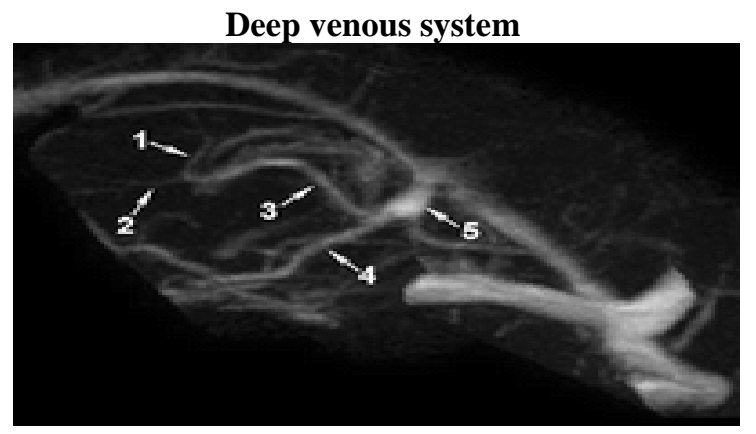

1. Thalamostriate vein

2. septal vein

3. internal cerebral vein

4. basal vein of Rosenthal

5. vein of Galen 
Basal dural sinuses

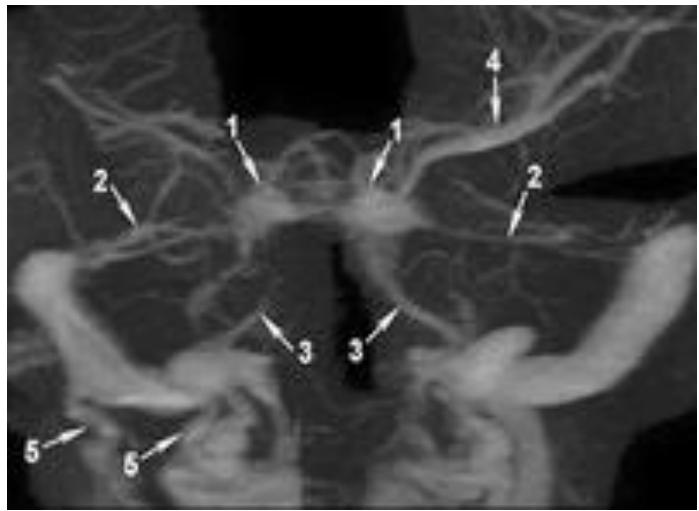

1 Cavernous sinus

2 Superior petrosal sinus

3 Inferior petrosal sinus

4 Superficial middle cerebral vein

5 Emissary and occipital venous plexus complex

TECHNIQUE OF ACQUIRING CONTRAST MR VENOGRAM

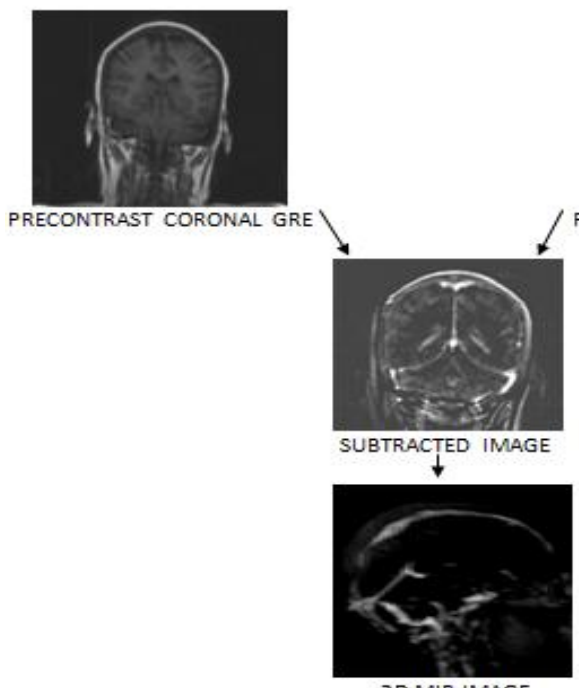

3D MIP IMAGE
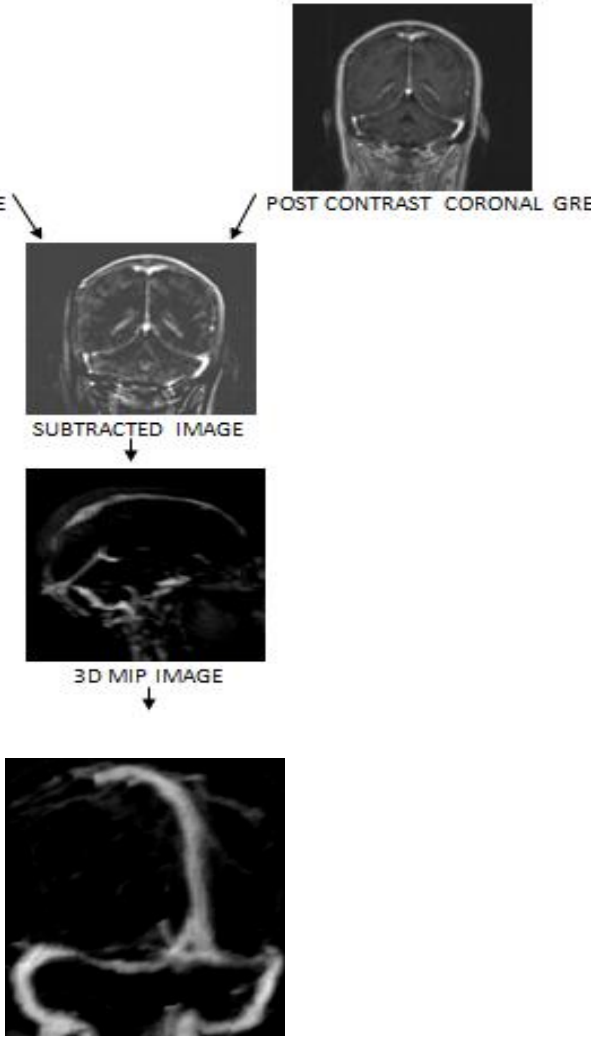

Segmented MIP Image

2D TOF MR Venogram Vs Contrast MR Venogram 

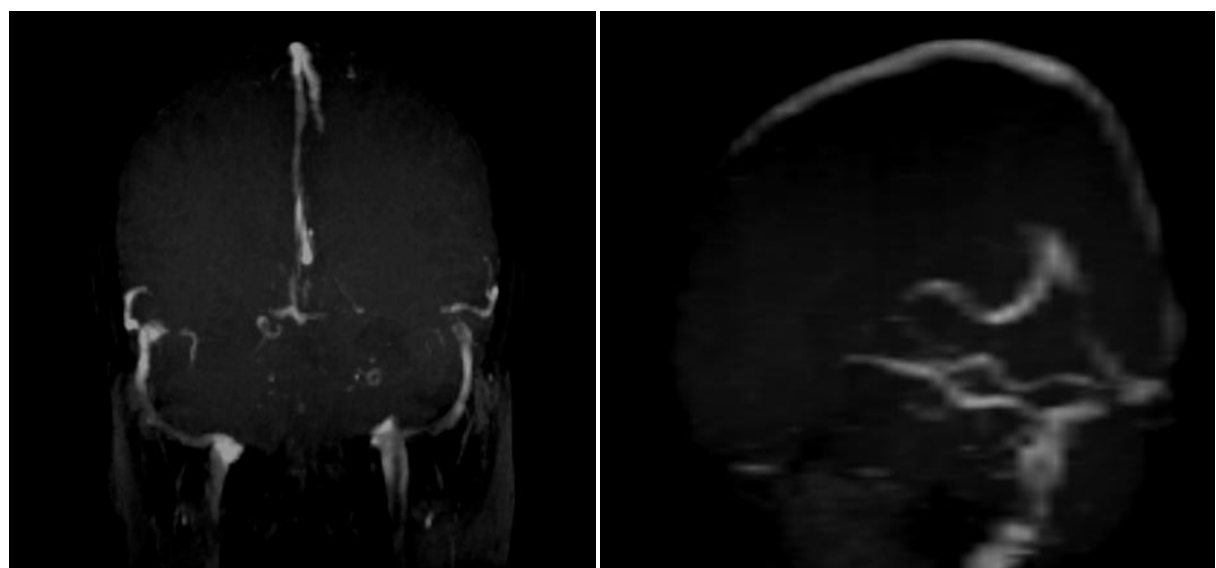

\section{D TOF MRV in transverse plane acquisition}

2D TOF MRV in coronal plane acquisition

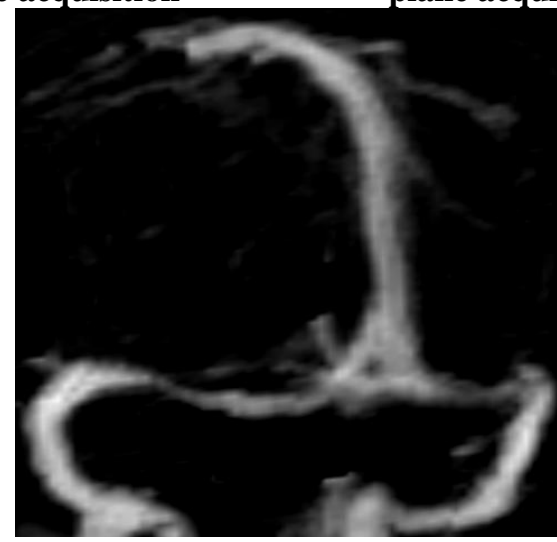

Contrast Enhanced MR Venogram

2D TOF MR venogram shows artifactual signal loss due do in plane flow error Slow flow, turbulent flow. Contrast enhanced venogram shows luminal diameter correctly as this is flow insensitive. The above example shows bilateral signal loss in both transverse sinus due to slow flow near the site of stenosis, whereas contrast MR venogram shows narrowed lumen as this is insensitive to slow flow

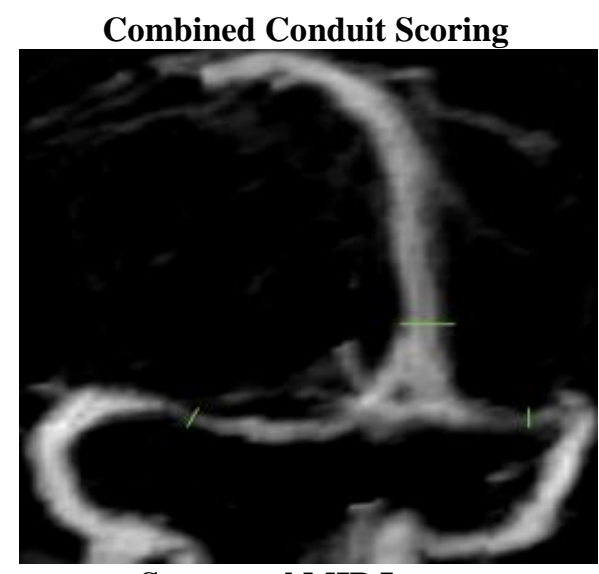

Segmental MIP Image

Distal luminal diameter of superior sagittal sinus compared with diameter of narrowed part of sigmoidtransverse conduit

\section{Grading}

0 - Discontinuity (gap) or aplastic segment;

1 Severe stenosis [less than $25 \%$ patent lumen ]

2 Moderate stenosis (25-50\% patent lumen ) 
3 Mild stenosis (50-75\% patent lumen); and

4 No significant stenosis (75-100\% patent lumen).

- Total score is 8 .

- when the score is less than 5 we consider the score significant and positive for intracranial hypertension

\section{Case -1}

38 years old female was referred for MR Venogram with the complaints of headache and blurring of vision . Fundoscopy showed bilateral papilledema . Her csf opening opening pressure which was known later in the study is $330 \mathrm{~mm}$ of water

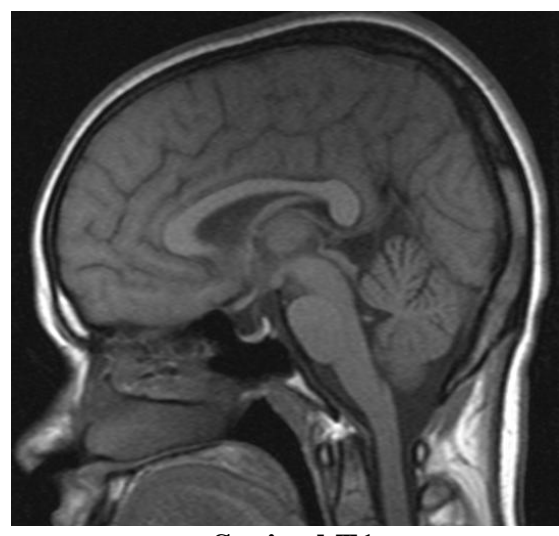

Sagittal T1

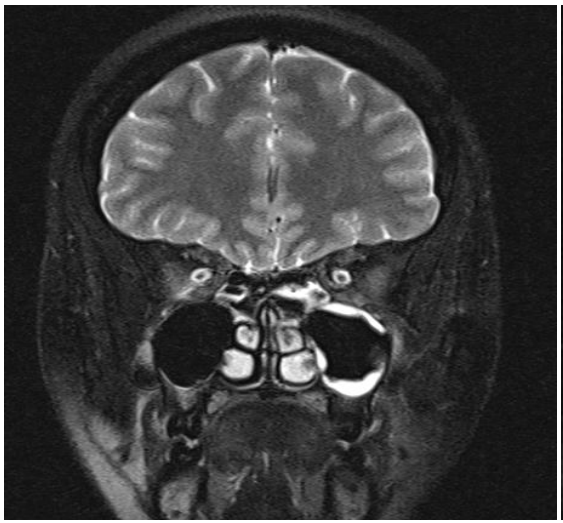

Coronal T2

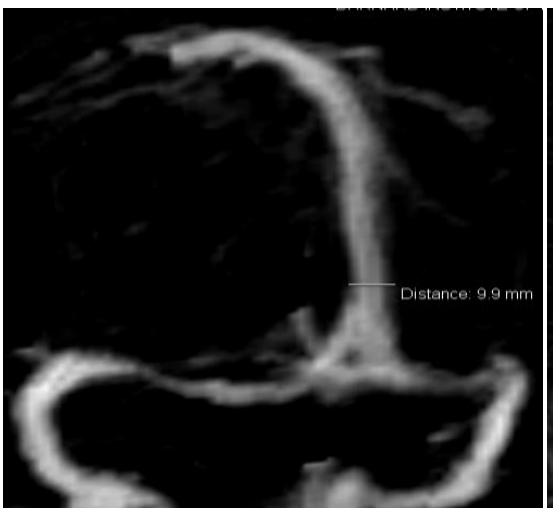

2D TOF MRV

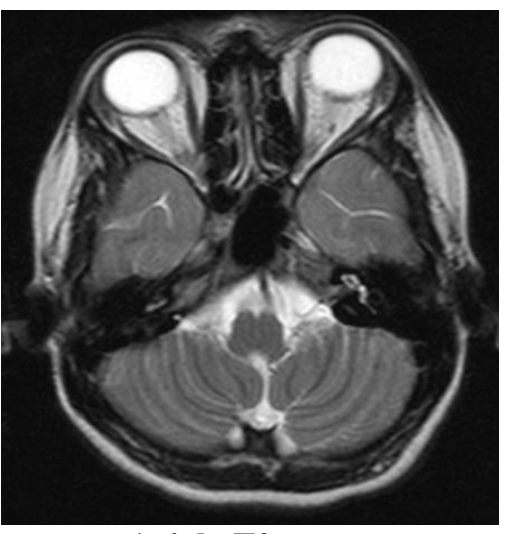

Axial T2

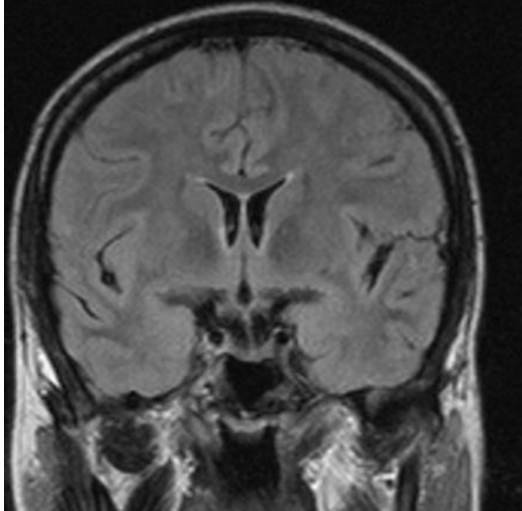

Flair Coronal

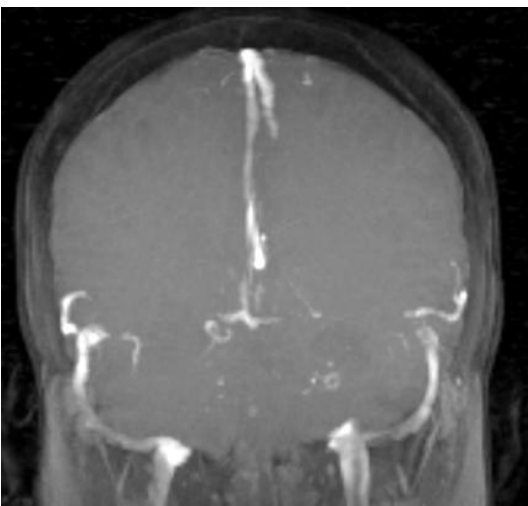

Contrast MRV Posterior 


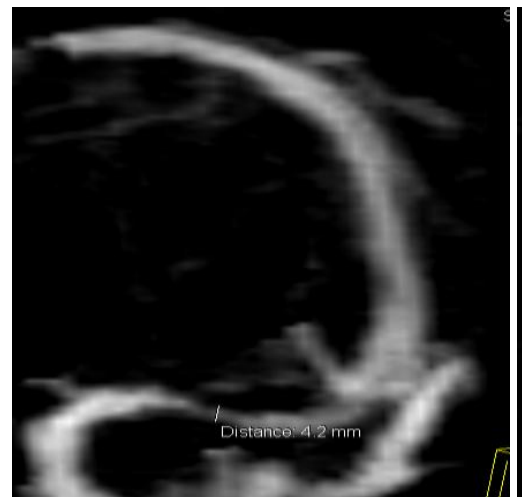

Contrast MRVenogram Left Oblique

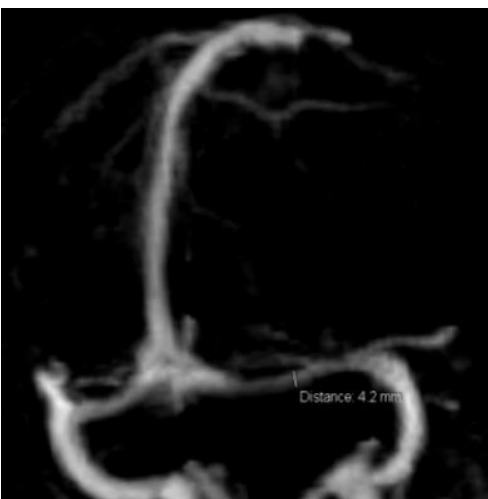

Contrast MRvenogram

Right Oblique

There is partial empty sella noted in T1 sagittal image, no obvious flattening of posterior sclera in $\mathrm{T} 2$ axial image, T2coronal image shows prominent perioptic space, coronal FLAIR image shows parial empty sella. 2D TOF MR venogram shows bilateral transverse sinus signal gap with a score of $0 / 8$.contrast enhanced venogram shows there is some patent lumen bilaterally which was shown as a signal gap in 2D TOF MRV The score using contrast MRvenogram is $4 / 8$ suggestive of significant stenosis

\section{Case 2}

50 years old lakshmi referred from department of neurology with the complaints of chronic head ache for 6 months and eye pain for 2 months and transient visual disturbances, she had bilateral papilledema. Her csf opening pressure which was known later in the study was $310 \mathrm{~mm}$ of water

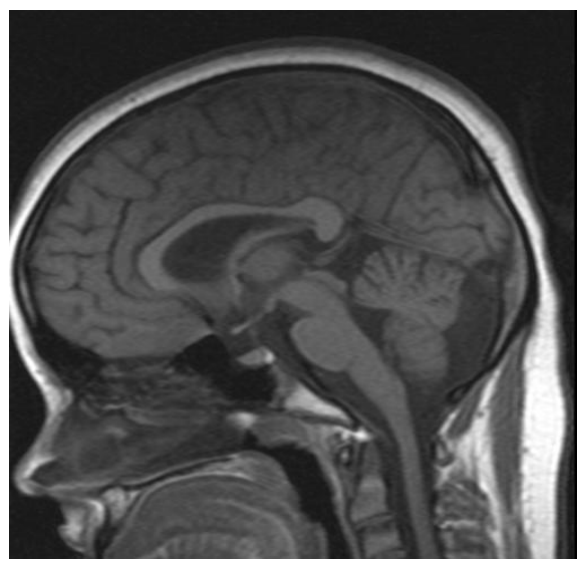

Sagittal T1

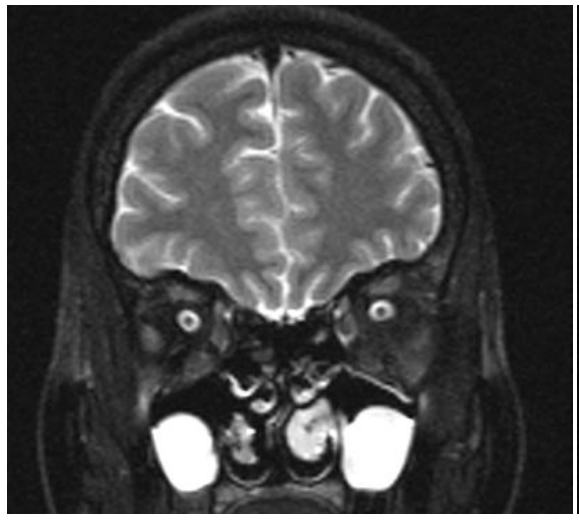

Coronal T2

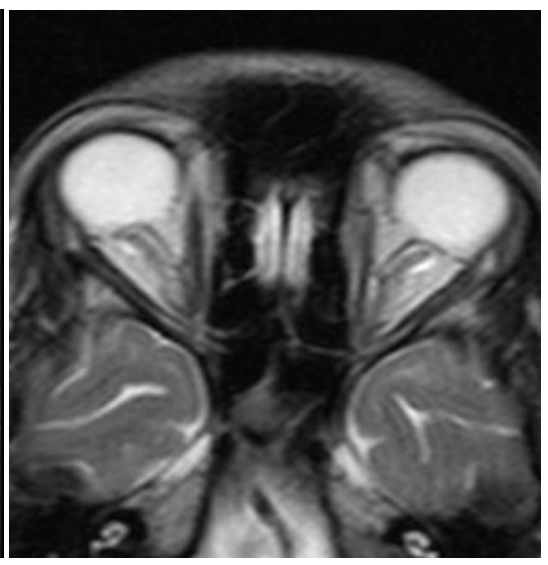

Coronal T2

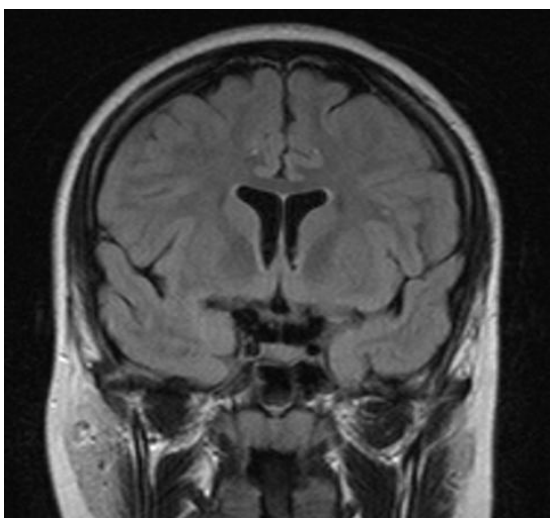

Flair Coronal 


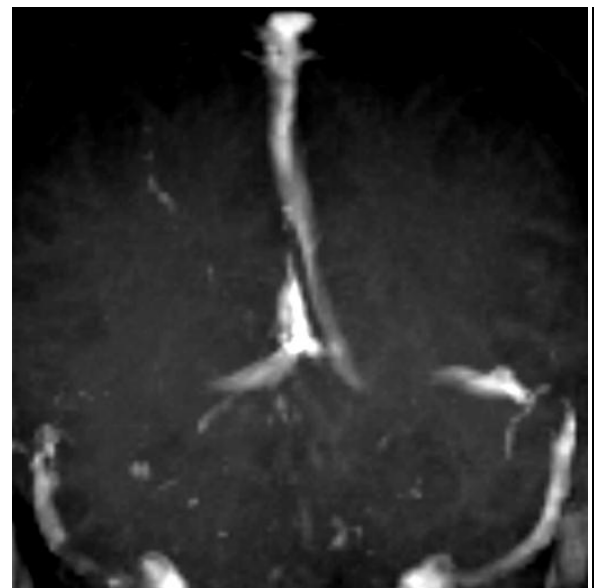

2D TOF MR Venogram

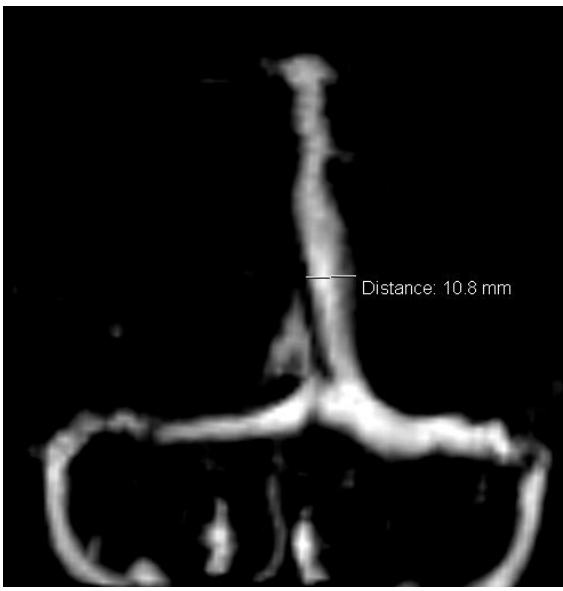

Contrast MR Venogram

\section{Posterior}

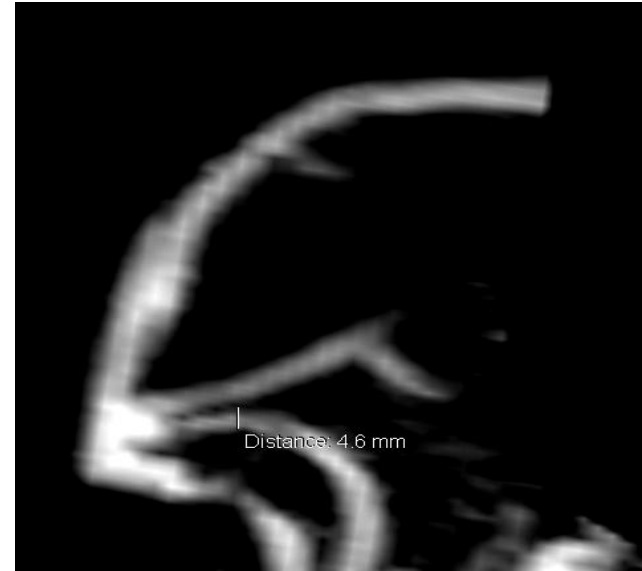

Right Oblique Contrast MRV

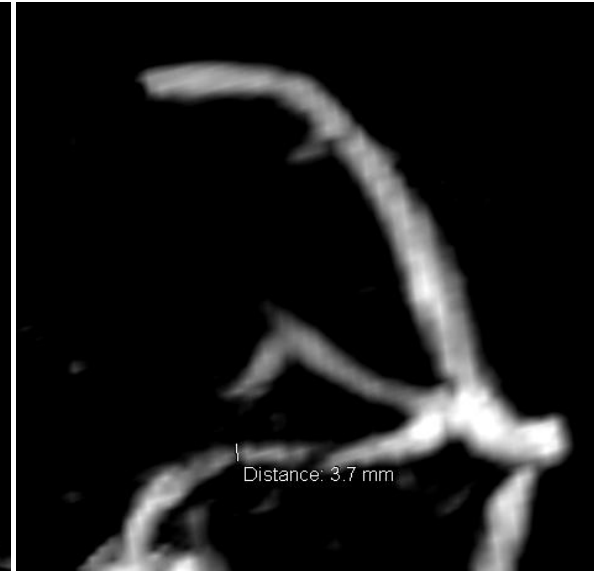

Left Oblique Contrast MRV

Sagittal T1 image shows normal pituitary,axial T2 shows flattening of posterior sclera and tortuous optic nerve sheath, coronal T2 shows prominent perioptic space, coronal FLAIR image shows normal pituitary. 2D TOF MR venogram shows bilateral signal gaps suggesting a score of 0/8 . contrast MR venogram shows patent flow in the signal gaps but with narrowing and with a score of $4 / 8$, hence this case is a true positive case

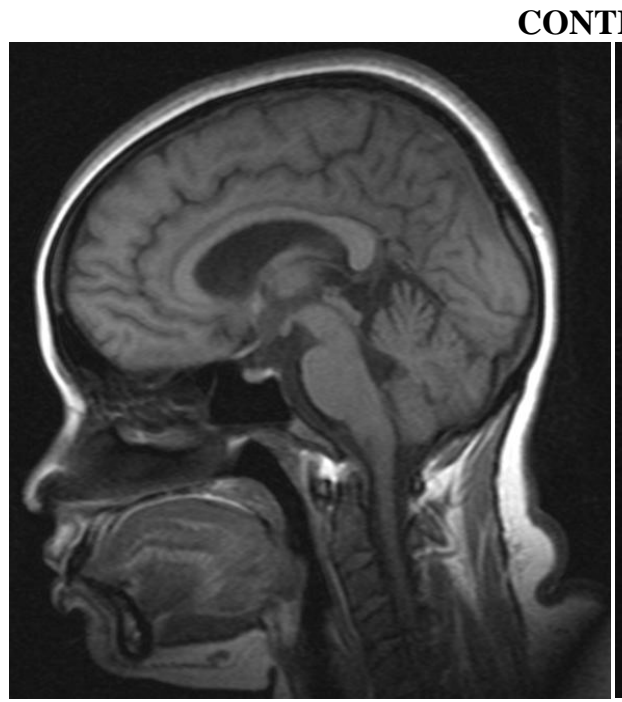

Sagittal T1

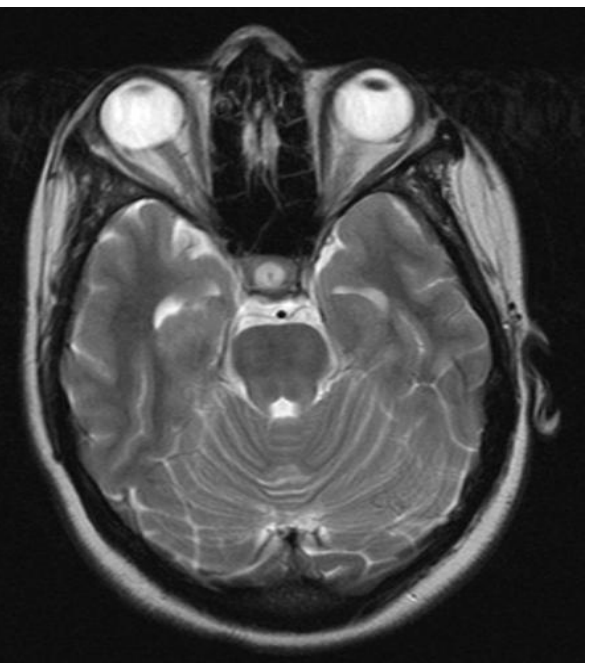

Axial T2 


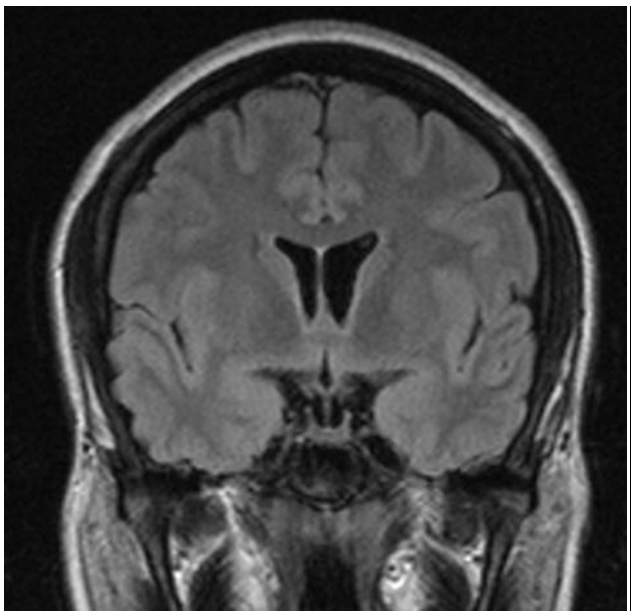

Flair Coronal

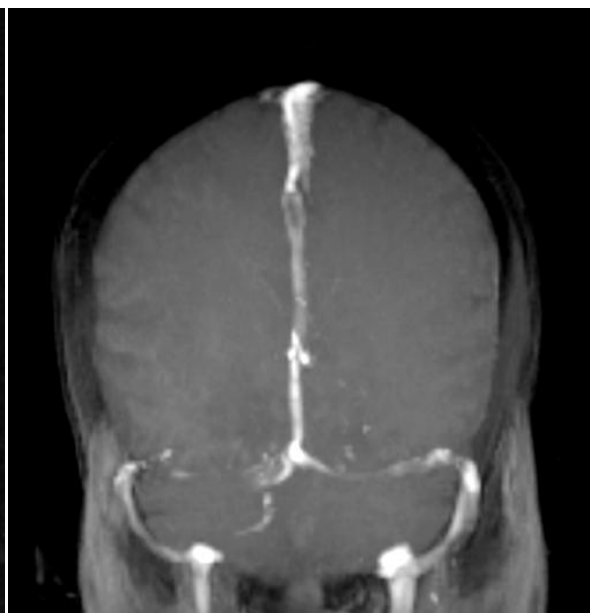

2D TOF MR Venogram

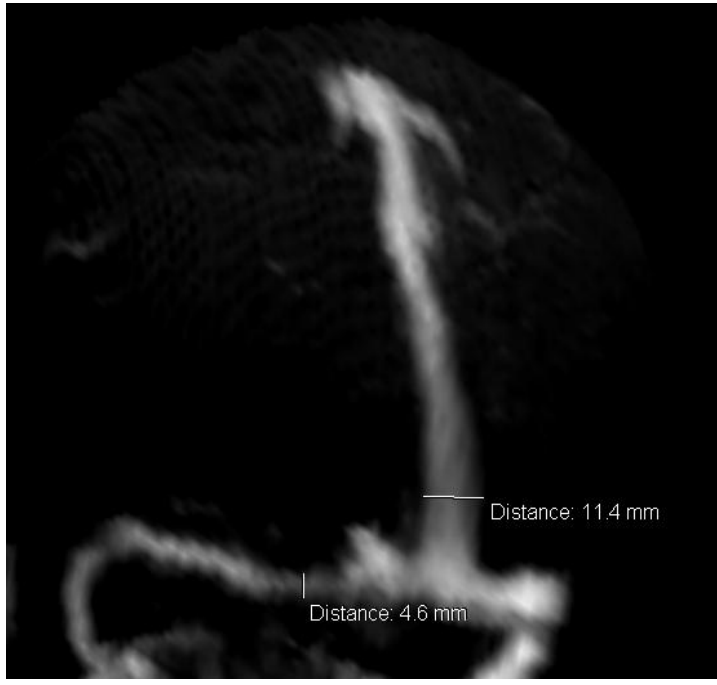

Contrast MR Venogram

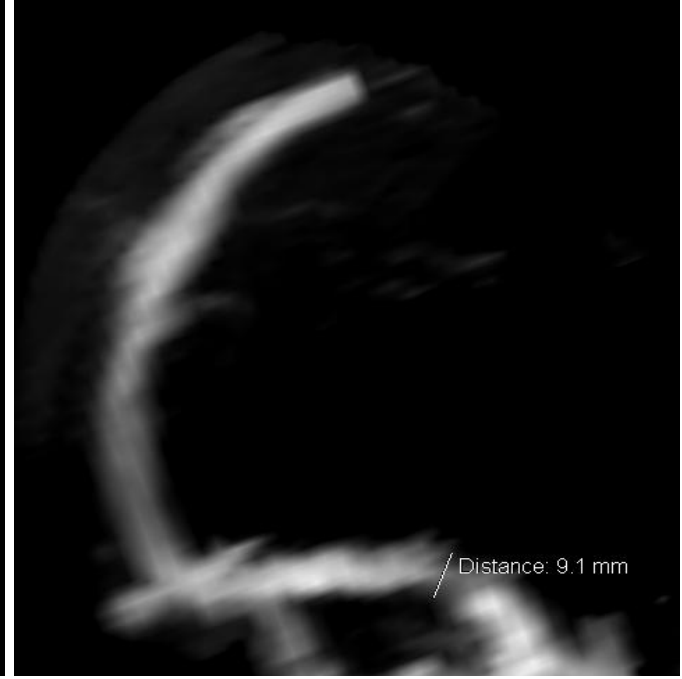

Contrast MR Venogram

37 years old female with complaints of chronic headache, no papilledema with csf pressure of $140 \mathrm{~mm}$ of water known later in the study . MRI reveals no features of idiopathic intracranial hypertension, 2D TOF MRV shows bilateral significant narrowing with flow gap on right side with a score of 1 , contrast enhanced MR venogram shows combined conduit score of 6 In this case 2D TOF MRV shows false positive value, whereas contrast enhanced MRV shows score more than 5 suggesting normal study 